\title{
Managing Knowledge in Urban Planning: Can Memory Support Systems Help?
}

\author{
Adele Celino, Grazia Concilio, and Anna De Liddo \\ Dipartimento di Architettura e Urbanistica Politecnico di Bari, Italy, [a.celino, g.concilio, \\ a.deliddo]@poliba.it
}

\begin{abstract}
When dealing with environmental plans, participation is considered crucial but hard work still has to be done in order to make participatory knowledge really operative. Tools and approaches to knowledge management are required that make participatory knowledge, which is produced, shared, and used along the planning action, available to the action itself. Starting from an experience of participatory planning in the context of Torre Guaceto natural Park, authors first reflect on the possible meaning of knowledge management in the planning process; secondly authors envisage the relevance of memory support systems in such processes as means to capture the argumentation chains which, explaining the action, are produced along the action and supporting it. Finally the paper presents the first results of a research project aiming at developing a memory support system dedicated to the Torre Guaceto Park Agency.
\end{abstract}

Keywords: Participatory knowledge management, Memory support, Argumentation, Long-term plans

\section{Introduction}

Participatory planning processes ask for and handle with knowledge collaboratively generated and validated. This knowledge is multiple (it comes from different peoples, sources and uses), plural (it is expression of different people needs and try to accomplish different tasks) and evolving (it changes in terms of time, space, social context settings and interpretations).

When dealing with environmental plans, participation is considered crucial but hard work still has to be done in order to make participatory knowledge really operative. More then in other domains, the need to keep trace of the assumptions, values, experiences, conversations, and decisions as they evolve along time is mainly oriented to enable reflection for the action-oriented plans development. Tools and approaches to knowledge management are required that make 
participatory knowledge, which is produced, shared, and used along the planning action, available to the action itself.

The paper argues the need to combine the concept of knowledge management with the concept of organizational memory in order to represent, take trace, and give reason to this knowledge process.

The paper starts from an experience of participatory planning in the context of Torre Guaceto natural Park, and represents it as just one of many planning stories where the ability to monitor and manage knowledge in participatory planning action is crucial for the effectiveness of the action itself. Following the reflections stimulated by that story, the authors first reflect on the possible meaning of knowledge management in the planning process, secondly envisage the relevance of memory support systems in such processes as means to capture the argumentation chains which, explaining the action, are produced along the action and supporting it. The perspective of "memory support systems" is discussed as an opportunity to orient knowledge management to action in participatory planning processes.

Finally the paper presents the first results of a research project aiming at developing a memory support system dedicated to the Torre Guaceto Park Agency.

\section{The story: Planning the Torre Guaceto wetland}

This case considers the activities carried out by the Park Agency of the Torre Guaceto wetland and the results obtained in its planning activity (Celino and Concilio, 2006a). Our analysis mainly focussed on the agency ability to reinterpret its planning tasks out of institutional protocols and to develop with the local community a communication framework, rather different from what it usually is in Italy. Since the very beginning of its activity the Park Agency approached participation practices conceiving participation not as a pre-structured planning protocol or simply one of the several activities of the planning process. Participants could not be captured into the planning process and simply managed as additional cognitive inputs. Two main conditions affected and still affect the interaction between the Park Agency and the local community.

1. The most recurring task for the Park Agency of Torre Guaceto is mainly oriented to plan and regulate in the area both agricultural uses and practices which are directly and heavily affecting the economic revenue of agricultural activities; discussions on the necessary modification of the agricultural practices could not avoid conflicts thus making interaction deeply lacking in communication and/or mutual learning and understanding.

2. A large number of land owners characterizes the area each representing a potential participant to be involved in the planning process. They are not 
only strong individual actors, due to their deep interest in the productivity of their activities; they also represent, all together, a rooted community whose communication dynamics gives shape to a complex and compact network. This network is difficult to be entered and cannot be summarised by one or two of its representatives.

The Park Agency developed the ability to act within the community as a part of it. It initially shaped its communication skills coherently with the communication mechanisms of the community network and subsequently, together with it, started to evolve. Coherently with the dynamics just described, a planning path out of codified protocols of the Park Agency can be outlined when looking at the Torre Guaceto activities.

The story we want to tell refers to the decision making process related to a specific portion of the Land Use Norms (part of the Reserve Spatial Management Plan): prescriptions for biological olive tree cultivation and olive oil production (Norms for Olive Oil Production, NOOP).

Constraints imposed onto land use practices usually activate strong reactions of individuals affected by the constraints. Also in Torre Guacteo deep aversion to the first version of that NOOP was given by the local agricultural community. Subsequently, new different versions of the NOOP, always less restrictive, have been proposed for the park area but no agreement with the agricultural community has been reached and norm, as means for innovating agricultural practices, has been temporarily abandoned.

A second version of the NOOP has been developed to restart the discussion among the Park Agency and the Torre Guaceto Agricultural Community each supported by their own consultants. In this second participatory phase the Park Agency tried to involve in the process all the cognitive actors recognized and accepted by the agricultural community thus shaping a new participatory environment characterized by knowledge flows, cognitions and actors different form those involved in the previous phase. Still, and similarly to the previous phase, collective discussions were organized, in the form of forum, in order to give rise to collaborative interactions. Also this second version of the norms has been deeply opposed since, may be, it was not considered by the agricultural community a product of its own reflection and decision.

Starting from an analysis of the agricultural community needs and requirements and using funds of an INTERREG project for supporting innovative agricultural practices in wetlands, a new communication protocol, out of the formal/institutional participation protocols for norms and plan adoption, has been activated. The new communication protocol involved the agricultural community in developing and testing a new practice for olive oil production: the agricultural community become the privileged actor of an experimental agricultural practice mainly due to its experience and practice.

A fourth version of the NOOP is currently being developed in form of Regulation of the Producers Association of Biological Olive Oil in Torre Guaceto 
and it is more restrictive than the first version of the NOOP elaborated by the park agency.

This story shows some elements which we consider crucial referring to the issue of making participatory knowledge operational for the decision.

Both the Agency and the Agricultural Community have shown cognitive openness: their availability to be involved in regional, national or international projects gave them the economical opportunities to start experimentation to find out protocols for innovative agricultural practices. This initial openness is now transformed: neither the Park Agency nor the agricultural community present solutions to each other. They are always looking for new practices making their productions no more impacting the wetland and no solutions is considered as such unless it is the result of a collaborative cognitive effort: knowledge is managed not to defend predetermined solutions bur rather to collaboratively create new ones.

The Park Agency continuously reframes its tasks within the area and has identified a new role for itself in park management. Within the general goal of guaranteeing the re-naturalization of the wetland and the sustainability of the surrounding areas, the Park Agency became a "process interpreter": its effort is no longer oriented to attract cognitive resources into a predetermined planning process, it rather observes the community mechanisms from its inside (it is now a component of that community, it behaves and is considered as in charge of some of the strategic community activities) and, contemporarily, from outside (it keeps its institutional managerial and monitoring tasks) and is able to continuously reshape the planning process, i.e. the action. In this way planning action is no longer the result of a pre-designed protocol, it is an emerging process which can be described by the interpretation of the continuous acting and deciding of the community and the Park Agency as a whole.

New agricultural practices have been tested and implemented looking for products which traditionally grown in that area although with a lower productivity. They are not completely new practices: they are the result of a creative search combining the re-use of traditional cultivar with modern agricultural techniques and transformation processes. Looking for possible solutions becomes a creative process based on cognitive collaboration. The enlarged community (Park Agency included) behaves like a pulsating entity: while searching solutions, it incorporates new components or, depending on the problem at hand, becomes part of other communities then returning to its previous condition although not unchanged. The modification required for an effective search did not only added new and operative knowledge, it rather represents a mode developed by the community to develop and manage knowledge throughout their participatory planning action: the planning action shapes itself around knowledge and its dynamics. 


\section{Managing knowledge in participatory planning environments}

The story of Torre Guaceto represents just one of many planning stories where the ability to monitor and manage knowledge in participatory planning action is crucial for the effectiveness of the action itself.

Although participation is considered fundamental in environmental planning activities (Tress and Tress, 2003; Kangas and Store, 2003; Mostert, 2003; Pellizoni, 2003), stories like this one highlight the crucial role of cognitions being produced, shared and used throughout such processes. The construction of environmental plans cannot be considered a mere addition, analysis and assessment of information and data: the construction of environmental plans is more and more intended as a social activity producing highly cognitive visions able to guide collective action and make it converge.

When building plans within participative processes, new methodologies and approaches for knowledge management are required since predictive knowledge needs either direct approaches to knowledge communication and exchange (vis-àvis meeting, web-based discussions) or relational approaches for social and institutional arenas management (communication, cooperation network management, public relations) (Jasper et al., 2004).

In the last years, a great effort has been carried out on the characterization of knowledge produced, shared and made operatively available during participative processes for plans development (consider for example the great discussion on lay and local knowledge); it is a distributed knowledge, scarcely structured, nonformalized and/or hard to formalize, organized and transferable in very different and particular forms (stories, traditions, practices, life styles, ...) difficult to acquire and, most relevant, difficult to manage within traditional knowledge management approaches.

Jasper, Banthien and Mayers-Ries (2004) introduced the concept of Participatory Knowledge Management (PKM) that is a knowledge management able to consider "soft facts of knowledge such as structural or cultural behaviour patterns, perception frames, values and opinions, implicit, practical and local knowledge" (Jasper et al., 2004: 69).

The problem of participatory knowledge management shows its peculiarity in both the specificity of involved cognitions and the modalities through which that knowledge is explicated, generated, and archived; it is a knowledge which:

- is multi- o trans-disciplinary,

- is highly flexible and reflexive,

- is produced in a variety of sites (formal or informal) and/or in virtual/ephemeral networks,

- needs new modes for quality control (attention has been recently paid to the knowledge assessment issue; EEA, 2001; Risbey et al., 2005).

Such knowledge is generated in action (Hage et al., 2006), and partially revealed and/or explicated only in action. Therefore action becomes the space in which modes for participatory knowledge management have to be defined and 
implemented. Obviously it is not possible to determine a priori such modes but it is possible to consider some very general rules, taking into account the peculiarity of participatory knowledge together with its strong relation to action.

This close relationship between knowledge management and action becomes crucial in participative processes for environmental planning: in such processes it is necessary to consider knowledge management as an activity able to give structure to action.

\section{Memory support systems: What perspectives}

As above remarked, the construction of environmental plans must be intended as a social activity in which evolving plans are (judged) able to guide the participatory action and make it converge. In such cases, the knowledge (often tacit) coming from participative processes could be considered as a value and utility only when it is able to stimulate collective, practical and more or less shared reflections (Celino and Concilio, 2006b).

The access to the knowledge developed by the decision making process during the process itself is particularly relevant for the plan development process since it strengths the support for reflection, enables re-experience considered fundamental mechanism for learning, (Celino and Concilio, 2006b) and widens the opportunities for decision making.

A plan developed in a participatory environment should focus not only on the substantive character of medium/long term visions but also, and especially, on the decisional system explaining those visions. Such a plan needs to evolve together with its decisional system and, consequently, to incorporate the continuous modifications that collective reflections produce on existing prospects, interpretations of the involved actors, and preliminary remarks explaining the decisions (Celino and Concilio, 2006b).

The need to explore the concept of organizational memory in participatory planning experiences starts from the stance to consider information and knowledge (used and produced in these processes) not as passive records to be stored in a repository but as dynamic contents living and changing along the time and coherently with the organization evolutions. It is important not only capturing and storing the history of the decision making process but also making it accessible for further and continuous interpretation and exploration along the process itself and by all the members of the organization, i.e. the stakeholders involved in building and using that memory day by day. As Fischer pointed out "organizational memory must be: i. extended and updated as it is used to support work practices, ii. continually reorganized to integrate new information and new concerns, and iii. serve work by making stored information relevant to the new task at hand" (Fischer, 2001:353). 
In environmental planning, capturing the Decision Rationale (DR) underlying the process itself and making it available to participants (Karacapilidis and Papadias, 2001; Santos et al., 1997; Alvarado et al., 2005) could be considered functional to the need to mediate between the short-term organizational memory and the long-term organizational memory.

Long-term organizational memory refers to those structures and contents of organizational memory being stable like values, principles, cognitions shared throughout the planning organization; short-term organizational memory represents essentially working memory (Miyake and Shah, 1999; Baddeley, 1986); deeply related to a specific decision making process it is an evolving entity supplying contents and knowledge to long-term memory throughout the DR considered as an intermediate memory and a stepping stone towards long-term organizational memory.

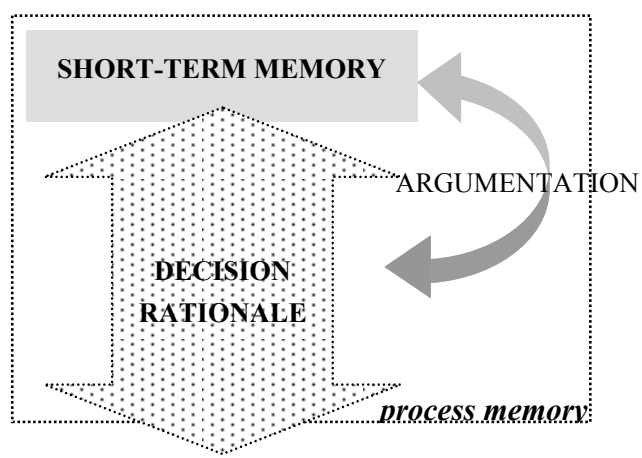

LONG-TERM MEMORY

Figure 1: The mediation role of Decision Rationale

Argumentation becomes crucial. It has a double role both in: i. making DR explicit, and ii. explaining the modifications of the short-term memory (or working memory) along (Shum, 1991; 1996) such modification process as a sequence of cognitive transactions (Wegner, 1995; Zeleny, 1989; Brauner and Becker, 2006) from one version of plan to the subsequent.

Our interest is not only in knowledge in itself but also in the underlying context of knowledge and in the process that created that context of knowledge at the time it is created (Miyake and Shah, 1999). Information systems, supporting such processes, need to be developed having a content repository and a context repository, both structured and organized in a process memory. In these systems argumentation would represent not only a relevant component of the DR (Tweed, 1998; Celino and Concilio, 2006b) but also the power engine of the plan evolution. Therefore, the process memory, referring to the tracing of the DR, has to include both the knowledge evolution and also the argumentative base explaining such evolution. 
In order to consider the temporal dimension of both the plan generation process and the plan itself, ICT systems should support the dynamic representation of the memory of the organization involved; indeed, such representation of the memory has to be considered a tool to "store" and exchange knowledge but also a mean:

i. to make explicit (and then visible to the users and system manager) the cognitive conditions and the argumentations generating the transactions from one version of the plan to the subsequent and

ii. to explore the operability of the current plan.

Starting from the model briefly described above, we are exploring the opportunity to represent dynamically the process memory within ICT environments by enabling the development of what we defined process-scenario that is a scenario which evolves together with its related decision making contexts and with the action itself (Celino and Concilio, 2006b). In a sense, the processscenario includes the process-memory (the "Design Rationale as the reasons behind spatial planning decisions) and the short-term memory (the current version of the plan).

The short-term memory has got a deeper operative role: it represents the support of the operative environment where decisions are made and, at the generic time $t$, contains the current version of the environmental plan and the whole cognitive content developed, acquired and created referring to that version This last would promote shared understanding at time $t$ about the context situation but does not have long-term value; it is just part of the process-scenario.

Coherently with the considerations above, the Organizational Memory System we are developing allows: i. to record and assist the knowledge generation and management (expressed in several media: graphs, images, texts, video, audio, etc.), ii. to keep trace of the history of decision, giving a structure to the memory of the complex "ephemeral" organization (Lanzara, 1983) emerging all around the participatory planning process, iii. to extract from history specific or new contents which are, or become during the process, "the focus of discussions and actions"; finally, iv. to trace the contents evolution and modifications along the process.

The Organizational Memory System allows users to create new knowledge rather than restricting them to the consumption of existing knowledge. Following the philosophy of meta-design (Fischer, et al., 2004), the system aims to support a reflective community in a collaborative design process; in such a way, the system is an open system that users can modify and evolve (Celino and Concilio, 2005).

\section{The architecture of the memory support system for Torre Guaceto}

In the following paragraph we present an experiment we are carrying out in Torre Guaceto in order to support a new starting project aiming at producing tomatoes in a biological perspective. This new project aims at implementing a memory support 
system assisting the community while developing new cultivation strategies and practices and is supposed to involve mainly the same community of the Torre Guaceto story told above. The system aims at tracking the memory of the decision making process thus making the knowledge produced along the action available for the action itself. That group of cultivators, collaborating with the Park Agency, is now interested in expanding its activities and possibly widening the community; all this with the help and supervision of the Park Agency to which it now recognizes a coordination role.

Starting from the lesson learnt from the NOOP Torre Guaceto story and described in paragraph 2, we designed a system prototype that can support the Agency in:

a. describing and monitoring the planning process and its main decisional steps;

b. capturing actions and decisions of the community and the park agency as a whole;

c. showing the knowledge network dynamics;

d. structuring and representing meetings contents and results;

e. detecting the features of the emerging community.

In other words the system would help the Agency to monitor the process and then to build new knowledge to put into the action of the on-going work.

The Park Agency acting as participatory process tracer can develop and improve its ability to manage knowledge within action. Process tracing is here considered the activity of tracing the development of a plan, i.e. to build the process memory related to the plan development.

The question to unfold is: is a memory system the right tool to orient knowledge management to action?

We start from the base assumption that any kind of reasoning and reflection about both process and knowledge needs to consider several aspects of knowledge itself, which are highly context dependent (Ackermann, 1982). When this process is collaborative and knowledge intensive, like participatory planning processes are, the context is rapidly changing and deep reflections are needed in order: 1 . to distinguish in the process between changing and resistant features, and 2 . to interpret and make sense of what is happening. Observation, reflection and action often rely on personal participants' skills, in particular to their ability or practice to carry out effective actions in rapidly changing contexts. We argue that ICT tools devoted to memory tracing can offer a valuable support to combine eventual community skills with more systematic benefits coming from more structured process of memory exploration.

Memory building activities can bridge knowledge to action in three ways at least:

1) putting knowledge in multiple-contexts,

2) showing the effects of past actions in similar or different contexts,

3) understanding the reasons for that context to be.

By performing these activities the Memory Support System would enable: 
a. better-informed actions, based on multiple-context explorations and cross-temporal comparisons with other cases (other knowledge applied to the same action, or other actions derived from the same knowledge);

b. higher transparency and understanding of the scopes behind actions (exploring reasons behind decisions helps in understanding where the process is going and why, so that we can monitor and eventually change, on going, the process direction; this helps to better orient actions toward the goals of the actions themselves).

We designed a memory system focusing on the points 1 and 3 stated before: we tried to represent knowledge in multiple contexts and to trace the decisions rationale, that is to say explaining reasons behind decisions and actions.

The system is being developed in the Compendium (Bachler et al., 2003) environment. Compendium is a hypermedia and sense-making tool used as a Knowledge Management Tool to store, structure and represent dialogical contents. The dialogical contents are extracted from argumentative dialogues performed along the participatory planning activities. Members of the community discuss problems, negotiate resources and select alternatives in order to reach collaborative decisions and actions. This dialogical process is often unstructured but it is stored referring to the key descriptors of the process (actors, time, problem environment, relations to other discussions, ...) thus reducing the effect of interpretation and structuring effort needed to convert real discussions in what we define argumentative-discussions (Hitchcock, 2002). Argumentation needs to be recognized within contents but contents are pre-structured and can easily support the re-tracking of argumentation chains. Contents of dialogues are extracted from meeting recordings and/or from meeting notes obtained with dialogue mapping techniques (Conklin et al., 2001).

In order to reduce the influence of subjective interpretation, contents are stored according to key descriptors of process which are organized coherently with five dimensions of participatory planning processes: conceptual, social, spatial, temporal and causal. A Soft System Methodology has been used to test the use of the hypermedia environment as knowledge management tool to represent and manage deliberation in participatory planning processes. As a first step we defined a conceptual model of knowledge object taxonomy. We recognized and defined the aspects (memory objects) of memory that need or use to be recollected during a Participatory Planning Process. Memory object types are: knowledge claims, decisions, information, process details, social details, geographical details etc. Based on this analysis we defined the memory object taxonomy to tag the knowledge objects during the knowledge tracking. This taxonomy has been used as data collection framework to annotate and classify knowledge objects and then represent them in the hypermedia database. In the knowledge taxonomy five dimensions have been recognized as constitutive for participatory planning processes.

Actors: Being this process participatory a first constitutive dimension has been considered the social one: who are the actors and what they say? 
Time: Furthermore, because dealing with a process of deliberation and design, the time dimension has been considered key to contextualize contents to actions: when in the process something has been said?

Space: Moreover the spatial dimension is considered constitutive because managing spatial planning activities: often the statements need to be referred to geographical areas or to spatial objects mainly because they refer to spatial issues.

Concepts: The conceptual dimension is considered constitutive of to represent dialogues and deliberation: in which dialogical and argumentative content a statement has been raised? In which context of dicussion?

Rationale: Finally what we defined "causal" dimension refers to causal chains of arguments. This is considered a key dimension because it motivates decisions then offering a representation of the design rationale.

The five dimensional knowledge object taxonomy have been tested in this pilot project to represent the contents of the Torre Guaceto project. Results of the application and evaluation of the memory system will follow in order to confirm or revise the knowledge taxonomy as in a soft system methodology approach.

In the following picture we show one view of the system enabling the access to memory from one of these dimensions (the conceptual one), in particular the figure 2 shows an image of argumentative dialogue extracted from one of the community meeting.

The dialogues are structured with an Issue-Based Information System (IBIS). IBIS is an argumentation model distinguishing between issues, positions on these issues, and arguments pro and con these positions (Kunz and Rittel, 1970). The IBIS argumentative model is used to represent contents of the meetings and then additional contents is performed to show and explore the contents through the five dimensions one by one. Following the argumentative chain it is possible to observe roles, trust relationships and decisional steps. The system allows monitoring the meetings and memorizing the contents.

Another view is the social one; it mainly consists of a list of participants attending the meetings; starting from the icon of each participant it is possible to explore general information, institutional roles in the project, his/her own network of relationships, list of the actor's statements and indexes to the video replay of the meeting (in case the meeting was extracted from a video).

The final result is a multiple-knowledge repository, organized in content and context sub-repositories, in which every actor's statement can be explored according with its temporal, conceptual, spatial, social and causal-argumentative context. These multiple "views" on knowledge offer :i. a knowledge-base for further analysis and evaluation ii. a detailed and multiple contextualization of information and knowledge produced during the process and iii. the tracing of the decision rationale in form of argumentative chains explaining decisions. 


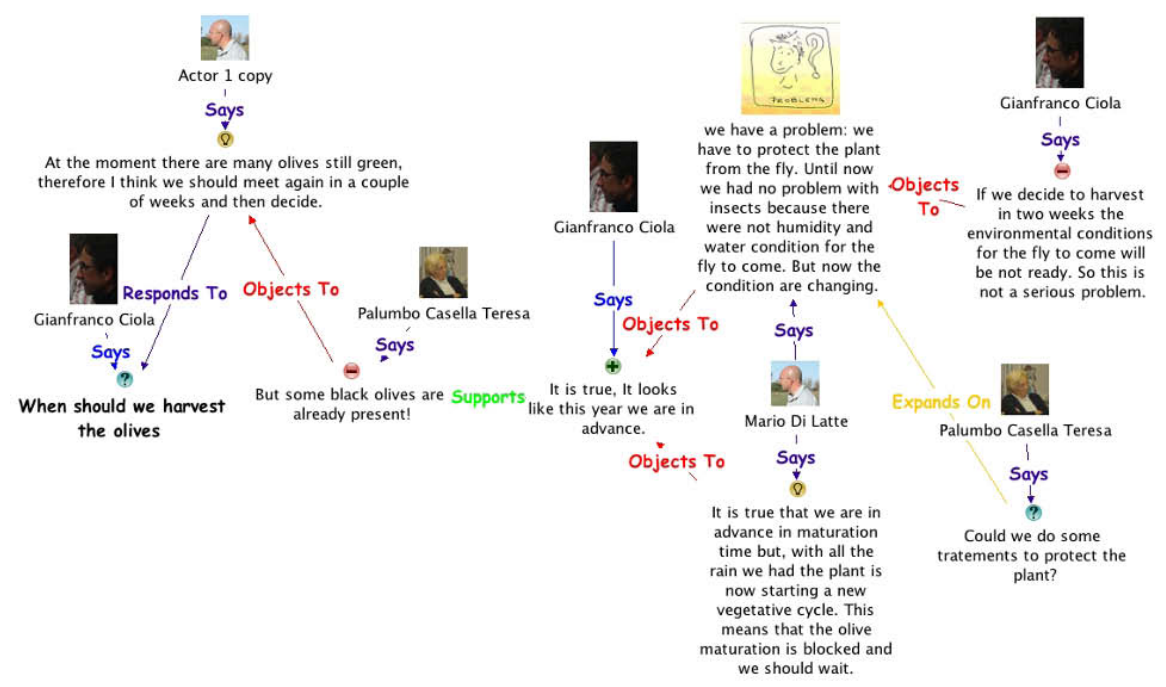

Figure 2. The conceptual view showing argumentative-discussions.

\section{Conclusions}

The paper explored the concept of organizational memory in participatory planning considering information and knowledge, used and produced in participatory processes, not as passive records but as dynamic contents living and changing along the time.

Since the ability to monitor and manage knowledge in participatory planning action is crucial for the effectiveness of the action itself, the paper proposes memory support systems as opportunities to orient knowledge management to action. In such systems the focus on the argumentation content is combined with the necessity to merge argumentation and environmental planning issues in a sort of memory, the process-memory, which is considered relevant for two main reasons: i. it supports effectively the environmental planning decision making process and; ii. it represents a sort of cognitive guide to orient action coherently with the indication contained in the environmental plan.

The memory system prototype we are developing for Torre Guaceto Park Agency stresses the tracing of the decision rationale; large effort still needs to be devoted to make this tracing more effective, less energy and time consuming and then consequently to produce contents which are available during the planning action so that these can be used from the community in order to support the action itself. The system is being developed to support the entire planning process by providing an integrated collaborative environment as structured space for participatory decision making. In such a collaborative environment knowledge is 
activated as a resource by the users who form relationships, act together, share and reflect collectively on their knowledge and beliefs.

At the moment using the system requires technical skills; both knowledge classification skills and technological training with the Knowledge Management tool are needed. Future research efforts will be devoted to complete the implementation of the memory system by improving functions oriented to the structuring and re-using the knowledge contents.

The reflections and the approach proposed in this paper derive from experiences and observations carried out in the environmental planning domain. It refers to plans development activities and, in particular, focuses on potentials of knowledge management in supporting the creation, management and use of evolving organizational memory in collaborative decision support systems. Our approach to KM focuses on a perspective in which workers as stakeholders create new knowledge as they carry out their work practices. Our goal is to enable innovative practices at a social level by supporting collaboration and communication. We see knowledge as an intrinsic aspect of collaborative practices production, in which stakeholders are integrating the knowledge they collaboratively construct into the (re)production of solutions and the practices themselves.

Generalizing, our reflections and approach could keep their validity in those domains where: i. decision making is characterized by collaboration and knowledge intensive interaction among stakeholder and ii. strategic planning activities are carried out in a futures visioning approach; ii. the dynamics of organizational memory is relevant for knowledge management and decision support.

Acknowledgements: The present contribution presents some results of a Research Work currently carried on in the Dipartimento di Architettura e Urbanistica of the Politecnico di Bari and funded by the Puglia Regional Administration: PE077 PARCHIAPERTI (POR 2000-2006).

\section{References}

Ackermann, R.. : Context Dependent Knowledge. Philosophy and Phenomenological Research, 42(3):425-433 (1982).

Alvarado, M. , Banares-Alcantara, R., and Trujillo, A.: Improving the Organizational Memory by recording decision making, rationale and team configuration. Journal of Petroleum Science and Engineering, 47:71-88 (2005).

Bachler, M., Buckingham Shum S., De Roure, D., Michaelides D., and Page, K.: Ontological mediation of meeting structure: Argumentation, annotation, and navigation. In: $1 \mathrm{st}$ International Workshop on Hypermedia and the Semantic Web. Nottingham, UK, 30th August (2003).

Baddeley, AD.: Working Memory, Oxford Univ. Press, Oxford (1986). 
Brauner, E., Becker, A.: Beyond Knowledge Sharing: The Management of Transactive Knowledge Systems, Knowledge and Process Management, 13(1):62-71 (2006).

Celino, A., Concilio, G.: Supporting collaborative learning in environmental scenario building through an argumentative system. Knowledge Management Research \& Practice, 4:240-249 (2006b).

Celino, A., Concilio, G.: E-Governance or environmental planning: perspectives of open content systems. In Dan Remenyi (ed.), Proceedings of the International Conference on egovernment, Academic Conferences Limited Reading, UK, pp. 89-99. (ISBN 1-905305-11-7) (2005).

Celino, A., Concilio, G.: Managing open contents for collaborative deliberation in environmental planning. In F. Malpica, A. Oropeza, J. Carrasquero, \& P. Howell, eds., Proceedings PISTA 2006. The 4th International Conference on Politics and Information Systems: Technologies and Applications, International Institute of Informatics and Systemics, pp.155-160 2006a.

Conklin, J., Selvin, A., Shum Buckingham, S., Sierhuis, M.: Facilitated hypertext for collective sensemaking: 15 years on from gibis. In: HYPERTEXT '01 Proceedings of the twelfth ACM conference on Hypertext and Hypermedia:123-124, New York, NY (2001).

EEA: Participatory integrated assessment methods: An assessment of their usefulness to the European Environmental Agency. Technical Report n.64. http://reports.eea.europa.eu/Technical_report_no_64/en/Technical_Report_64 (2001).

Fischer, G., Arias, E., Eden, H., Gorman, A., and Scharff, E.: Transcending the Individual Human Mind - Creating Shared Understanding through Collaborative Design. In J. Carroll (ed.) "Human Computer Interaction in the New Millennium", Addison-Wesley, pp. 347-372 (2001).

Fischer, G., Giaccardi, E., Ye, Y., Sutcliffe, A.G., and Mehandjiev, N.: Meta-design: A manifesto for end-user development, Communications of the ACM, volume 47, number 9, pp. 33-37 (2004).

Hage, M, Leroy, P, Willems, E.: Participatory Approaches in Governance and in Knowledge Production: What Makes the Difference? Working paper series 2006/3, Research Group Governance and Places, University of Nijmegen. http://www.ru.nl/contents/pages/141634/gapwp06-03.pdf. (2006).

Hitchcock, D.: The Practice of Argumentative Discussion. Argumentation, 16(3): 287-298 (2002).

Jaspers, M., Banthien, H., Mayer-Ries, J.: New forms of knowledge management in participatory foresight: The case of "Futur". Eu-us seminar: New technology foresight, forecasting \& assessment methods (Seville, May) (2004).

Kangas, J., Store, R.: Internet and teledemocracy in participatory planning of natural resources management. Landscape and Urban Planning, 62:89-101 (2003).

Karacapilidis, N., Papadias, D.: Computer supported argumentation and collaborative decision making: the HERMES system. Information Systems, 26:259-277 (2001).

Kunz, W., Rittel, HWJ.: Issues as elements of information systems. Technical Report WP-131, University of California, Berkeley (1970).

Lanzara, GF.: Ephemeral organizations in extreme environments: Emergence, strategy, extinction. Journal of Management Studies, 20(1):71-95 (1983).

Miyake, A., Shah, P.: Models of working memory: Mechanisms of active maintenance and executive control. Cambridge University Press, New York (1999).

Mostert, E.: The Challenge for Public Participation. Water Policy, 5:179-197 (2003).

Pellizoni, L.: Uncertainty and Participatory Democracy. Environmental Values, 12:195-224 (2003).

Risbey, J., Sluijs, J., Kloprogge, P., Ravetz, J., Funtowicz, S., Corral Quintana, S. Application of a checklist for quality assistance in environmental modelling to an energy model. Environmental Modeling \& Assessment, 10(1):63-79 (2005).

Santos, AC., Galina, SVR., Alves, AC. , Fagundes, LG.: Adapting a Decision Making Synchronous/Asynchronous Environment to a Distributed Hypermedia Concurrent 
Engineering System. In: 30th Hawaii International Conference on System Sciences, 6 to 10 January, Maui - New York. IEEE, 1: 686-694 (1997).

Shum, S.: Cognitive Dimensions of Design Rationale. In: Diaper D, Hammond NV (eds) People and Computers VI: Proceedings of HCI'91. Cambridge University Press, Cambridge, 331344 (1991).

Shum, S.: Analyzing the Usability of a Design Rationale Notation. In: Moran TP, Carroll JM (eds) Design Rationale: Concepts, Techniques, and Use. Lawrence Erlbaum Associates, Hillsdale, 185-215 (1996).

Tress, B., Tress, G.: Scenario visualisation for participatory landscape planning - a study from Denmark. Landscape and Urban Planning, 64:161-178 (2003).

Tweed, C.: Supporting Argumentation Practices in Urban Planning and Design. Comput., Environ. and Urban Systems, 22(4):351-363 (1998).

Wegner, DM.: A computer network model of human transactive memory. Social Cognition, 13:319-339 (1995).

Zeleny, M.: Cognitive equilibrium: a new paradigm of decision making?. Human Syst. Manage., 8: 185-188 (1989). 